\title{
Algunos aragonesismos del Atles Lingüístic de la Diòcesi de Tortosa (ALDT): una aproximación
}

\author{
Lluís GIMENO BETÍ \\ Departament de Filologia i Cultures Europees \\ Universitat Jaume I \\ llgimeno@fil.uji.es \\ Real Academia de Buenas Letras de Barcelona
}

\section{RESUMEN}

Este es el análisis léxico de algunos aragonesismos aparecidos en la zona dialectal de Tortosa dentro del territorio lingüístico catalán. El método de trabajo es analizar las palabras de una serie de diccionarios, catalán sobre todo occidental, con la incorporación de la documentación literaria y de su extensión geográfica.

Palabras clave: lengua catalana, lengua aragonesa, léxico románico, Tortosa

[Recibido, agosto 2013; aprobado, diciembre 2013]

Some Aragonese words in the Atlas Lingüistic de la Diòcesi de Tortosa (ALDT): an approach

\begin{abstract}
This is the lexical analysis of some aragonesisms appeared in the Tortosa dialectal area of the Catalan linguistic territory. The working method is to analyze the words from a series of dictionaries, especially Western Catalan, with the incorporation of literary documentation and its geographical extension.
\end{abstract}

Keywords: Catalan language, Aragonese language, romance lexis, Tortosa 


\section{Introducción}

El estudio del aragonés en su relación con los atlas lingüísticos es de gran importancia para el conocimiento del léxico de las lenguas catalana y española, y no es necesario insistir en ello por sabido. Hace ya muchos años, y en la línea que apuntábamos, Manuel Alvar daba muestras de su maestría publicando en la revista "Archivo de Filología Aragonesa" de Zaragoza un trabajo que titulaba "Léxico aragonés del 'ALC"1. Por otra parte, Germán Colón ha publicado, a lo largo de su dilatada carrera universitaria e investigadora, diversos trabajos que ponen en relación el aragonés con las lenguas hispánicas antes mencionadas; con ello, no se termina la lista de los filólogos que han puesto manos a la obra en el campo de la lingüística aragonesa, ni mucho menos. No obstante, hemos querido traer a colación estos dos grandes nombres de nuestra investigación filológica porque el presente trabajo debe mucho a la lectura de las obras de estos dos grandes investigadores, conocedores como pocos del aragonés y de su entorno lingüístico catalán y castellano.

\section{Relación entre lenguas}

La relación entre el castellano, el aragonés y el catalán viene de lejos. Por la parte oriental de la Península Ibérica, el aragonés ha sido una de las lenguas de adstrato del catalán -junto con el occitano por el norte- desde el primer momento del nacimiento de los respectivos romances; no será extraño, pues, que podamos hallar intercambios léxicos entre los dos romances desde los primeros textos literarios ${ }^{2}$ conocidos. El aragonés fue el primer diasistema lingüístico hispánico que se relacionó con el catalán. De ello, vinieron consecuencias lingüísticas que llegan hasta hoy, sin duda. En los estudios de hablas fronterizas se plantea a menudo el problema de la pertenencia de un determinado rasgo a la lengua $\mathrm{A}$ o a la lengua $\mathrm{B}$. Generalmente, se tiende a considerar, cuando el rasgo es característico de uno o de otro idioma, tal o tal préstamo. Por ejemplo, en el caso del aragonés o del catalán, se considera un aragonesismo del catalán si aquel rasgo es aragonés, y si es al revés, un catalanismo del aragonés. Así, podemos leer en la entrada Aragonesismos de la Gran Enciclopedia Aragonesa:

Poco o nada se han estudiado los aragonesismos en catalán; en la lengua literaria no hay apenas, pero en la región del Pallars existen influencias del aragonés, como la desinencia -iba en los pretéritos imperfectos de Indicativo de la segunda y tercera conjugación" (GEA, I, s.v.).

$\mathrm{Y}$ es que se olvida con demasiada frecuencia que en dialectos constitutivos de dos lenguas se producen fenómenos de continuum, es decir, que rasgos fonéticos, morfosintácticos o léxicos formados a partir de un fondo común latino, modificado por el substrato o por algún otro factor, son compartidos total o parcialmente por dos territorios lingüísticos adyacentes. En este sentido, Elcock (1937) al referirse al aragonés o al gascón, hablaba ya de casos de "simple concomitance", M. Alvar (1953) aludía a "límite de áreas léxicas", Pottier (1955) a "continuidad de área lingüística", Colón (1968) a “área léxica continua” y a "continuo léxico catalano-occitano", P. Bec (1968) a “interfé-

\footnotetext{
${ }^{1}$ Manuel Alvar, "Léxico aragonés del 'ALC", Archivo de Filología Aragonesa, VIII-IX, 211-238. Zaragoza, 1956-57.

${ }^{2}$ Por ejemplo, para el catalán, baste citar el Llibre dels Feyts de Jaume I, entre otros.
} 
rence primaire", y J. A. Frago (1977) a "comunidad de substrato", y a lo largo de este artículo se podrán comprobar estas afirmaciones porque en algún ejemplo parece que más propiamente del supuesto aragonesismo pueda tratarse de continuum.

\section{Objetivos}

El propósito del presente trabajo es analizar algunos aragonesismos vivos de la variedad diatópica tortosina ${ }^{3}$, sacados del Atles Lingüístic de la Diòcesi de Tortosa (ALDT), que forma parte del catalán occidental, dialecto más próximo geográficamente a las hablas aragonesas. Para ello, el método a seguir será exponer en qué obras (diccionarios, vocabularios, etc. catalanes, castellanos, aragoneses y en alguna ocasión románi$\cos$ ), en qué autores y desde cuándo se documentan, con las citas literarias si las hubiese, más la extensión geográfica que ocupan actualmente en catalán occidental junto a la etimología del término lingüístico.

\section{El campo semántico de la ganadería}

Un campo semántico brotado de aragonesismos en catalán occidental es el de la ganadería. Antes de la creación del Reino de Valencia, las comarcas de Castelló, ya desde hora muy temprana, fueron una de las zonas más estimadas por los ganados trashumantes de Aragón y a veces también de Castilla, por lo cual el intercambio ganadero entre las tierras de Teruel y de Cuenca y las castellonenses era muy frecuente puesto que los pastos de invierno para unos y los de verano para otros eran fundamentales para garantizar la estabilidad en el número de los ganados, y en definitiva la economía de base pecuaria como ha sido y continúa siendo la de estas tierras. Por otra parte, sabemos que una de les primeras gestiones de la incipiente Orden Militar de Montesa, creada en 1317, fue la regulación de la trashumancia ganadera por la importancia que tenia ya en el territorio. A las dehesas de Vallivana, Turmell y otros prados comunales de las comarcas valencianas septentrinales acudían los ganados aragoneses para pasar el invierno (Betí, 1927: 98). Otra actividad que nos demuestra abundantemente el intercambio entre las comarcas turolenses y las castellonenques son los pleitos por motivos de restricción de pastos ( $c f r$. Sánchez Almela, 1985: 316-324). Otro aspecto que acaba de remachar el clavo en ese sentido es la referencia a las vías pecuarias. El origen de estos caminos parece prerromano. Su trazado formaba y forma todavía actualmente una espesa red viaria importante tanto por su magnitud como por su significado histórico y, más importante aún que todo eso, para el intercambio lingüístico. El norte del País Valenciano és un espacio geográfico de gran entidad en cuanto a la existencia de estas vías. Las principales rutas son: a) El Maestrazgo-Bajo Aragón; b) Peñagolosa-La Plana-Bajo Aragón; c) Río Mijares y afluentes-Bajo Aragón; d) Los Ports-Bajo Aragón; e) el llamado 'Assagador dels romans' (la vía Augusta). Las tres primeras enlazan las comarcas castellonenses con Aragón y sólo la última lo hace con Cataluña de norte a sur hasta al límite de la comarca del Camp de Morvedre. No hará falta decir que estas rutas eran seguidas por caminos secundarios que enlazan las comarcas entre sí ${ }^{4}$.

\footnotetext{
${ }^{3}$ Vide, por ejemplo, Gimeno Betí, 1997: 14-17.

${ }^{4}$ Para una información más exhaustiva de las rutas ganaderas vide Obiol, 1989: 253-255, trabajo que incluye un mapa de la provincia de Castellón conteniendo las rutas, y Betí, 1927: 105-108.
} 


\section{Análisis de algunos vocablos}

El campo semántico a que nos referíamos más arriba viene representado en este trabajo por los vocablos azagador, ganado, ligallo y mallada, que pasamos a exponer seguidamente:

\subsection{AZAGADOR (cat. assagador/assegador):}

El ALDT recoge el término en el mapa núm. 203 'El camí ramader' (esp.'cañada', 'camino de cabaña'), bajo la variante assegador, que parece indicar la adaptación a la fonética del catalán occidental del aragonesismo azagador, aunque también exista en esta variedad diatópica catalana la forma más cercana a la aragonesa assagador. El Vocabulari del Maestrat (con grafia assagador) de García Girona define así el vocablo: "carrera de pas per a bestiars forasters a través dels camps i poblats, que sòl córrer entre dos parets de pedra seca. Assagadell a Tortosa, significa una cosa per l'estil, un erm voltat de paret seca per al bestiar. Azagador." Documentado en los repertorios lexicográficos valencianos: Escrig (1851: 106) açagador, hor 'azagador ó vereda, paso de ganado'; Llombart $(1887: 213,252)$ asagador 'camino carretero abierto á través de los campos para conducir las cosechas á las masadas, alquerías, barracas, etc. V. camí asagador, açagador, hor 'azagador. Vereda ó paso de ganado. Por ext., camino abierto á través de los campos para conducir cosechas y ganados á las masadas, alquerías, barracas, corrales, etc. V. asagador; Martí Gadea (1891: 330, 386) asagador 'v. açagador', açagador 'azagador: verada ó paso del ganado. Camino abierto á través de los campos para conducir cosechas y ganados'; Colomina (1991: 101) assegador 'camí per al pas del ramat; solia usar terrenys o llocs de planíssia, com ara barrancs o secans; generalment no era terra de ningú' (apud Ramon Llorens, Diccionario de Altea y sus cosas). Apelativo que ha dado muchos topónimos en Callosa, Benidorm, etc. Igualmente recogido en los diccionarios históricos catalanes: DCVB (II: 73) assagador 'camí obert per a servir al pas del bestiar o per traginar les collites'; DECLC (VII: 584) assagador 'camí tradicional de bestiar' al lado de assegador (Coromines duda si se debe de grafiar con $-a$ - o con $-e)^{5}$. Afirma que la palabra no está documentada durante el período medieval ni tampoco en ningún diccionario antiguo, puesto que el primer autor que lo recoge es Escrig (vide supra). Hay que decir, sin embargo, que M. Milián, en un artículo publicado en el BSCC del año 1927, incluye un documento, que muestra las relaciones entre Morella y Tortosa referido a la institución del ligallo, en que aparece nuestra palabra:

Item que $\cdot 1 \mathrm{~s}$ bestiars dels hòmens de la dita ciutat e de son terme puxen passar franchament $\mathrm{e}$ quítiament per los termes de Morella e de ses aldees camí caminant segons que bestiars ha acostumat caminar per los camins e açagadors que bestiar ha acostumat de pasar axí entés que passen per los camins e açagadors...” (Milián, 1927: 185).

El documento está datado en $1408^{6}$, por tanto en época medieval. Otro autor que también documentó el vocablo durante la edad media es mosén Betí: "fonch fitat e mudat

\footnotetext{
${ }^{5}$ El Onomasticon Cataloniae (II: 265) recoge la forma con -e- y remite, para todo tipos de aclaraciones, al DECLC (VII: 585-586). Dice el O.C: 'Assegador designant indrets on passa una carrerada'.

${ }^{6}$ La documentación valenciana más primeriza, por ahora, de la palabra es de 1384 en un documento de las comarcas centrales (vide Josep Martines, El valencià del segle XIX. Estudi lingüístic i edició del Diccionario
} 
lo camí cegador que ix del pla e va a la lloma del Rebollar" en un protocolo de un notario de Vilafranca de 1465. En este caso se nos presenta con la variante - e- y con apócope de la primera sílaba (Betí, 1927: 99). Es registrado por el Diccionari de la Llengua Catalana del IEC bajo la forma assegador.

Lo encontramos citado también en un document de 1418 del Archivo Municipal de Vilafamés (Plana Alta). Se trata de una sentencia arbitral sobre cuestiones diversas, entre las cuales se encuentra el uso de caminos y cañadas que utilizan los habitantes de Cabanes para la trashumancia ganadera al subir y bajar del puerto, y se les reconoce el derecho de uso estableciendo la reciprocidad para los de Vilafamés en término de aquella localidad. De este documento, existe una copia autentificada hecha en Cabanes el 16 de agosto de 1486:

Hoc encara se spere ésser qüestió per los bestiars que los vehins de Cabanes passen per terme de Vilafamés pugant al port, anant e devallant de aquell per qual camí deuen passar e per quals açagadors ni quant terme de camí o açagador los deu ésser donat dins terme de Vilafamés pugant e devallant (Rabassa-Díaz, 1995: 229-233)

Ulterius, com qüestió, contrast e debat se speràs a ésser entre lo dit honorable comanador e la universitat de Vilafameç de una part, e la universitat del loch de Cabanes de la altra part, per rahon e causa de les carreres e açagadors antichs e antigues, les quals de necessitat los hòmens vehins de Cabanes han aver als lurs bestiars com s'en pugen al port e.n devallen, e sia en rahó fundat que les dites carreres e açagadors antichs los sien atorguats per passar lurs bésties e bestiars... (ibidem)

Los quals camins e açagadors sien mollonats e fitats per dos bon hòmens de Vilafameç e dos bons hòmens de Cabanes elets a acscuna universitat... (ibidem)

E si per ventura alguns dels dits bestiars se exien del camí e.s desviaven de aquell, ço és lo bestiar de Vilafameç dins terme de Cabanes e lo bestiar de Cabanes dins terme de Vilafameç, que sobre açò no puxe ésser levada pena anant camí caminant cap avant lo dit bestiar, puys part del dit bestiar romandrà en lo dit camí o açaguador tro en tres o quatre cabeçes; e si tot lo dit bestiar serà fora del camí o açagador puixa ésser carnejat a set e quatorçe segons ús e stil antich. E semblant ampriu ajutgam als vehins del dit loch de Vilafameç qui ara són o per temps seran, que agen semblants abeuradors, peximents, açagadors e carreres si e segons han los del loch de Cabanes en terme de Vilafameç, per ço que entre aquells egualtat sia servada" (ibidem)

En el siglo XVI (1535) se documenta en la antigua Setena de Culla como Collet de l'Assagador de la Solana, y en este mismo documento, vuelve a ser citado en 1729 (Peraire, 1992: 120). Lo vemos citado de nuevo en 1566 en un pleito entre las localidades castellonenses de Cabanes y Vilafamés. Los litigios hacen referencia a diversas cuestiones, entre las cuales tenemos las cañadas, etc. (vide Rabassa-Díaz, 1995: 309).

Está documentado también en un texto denominado Regoneixença general de la Tinença de Culla de 1615: "Y més avant en lo assagador de la Tayola, en la costera trobaren una fita vella. Al sòl de la foya del mas, a un roure trobaren dos fites, la una endret de la altra" (Beltran Zaragozà, 1998, I: 127). En este mismo documento de 1615, lo volve-

Valenciano de Josep Pla i Costa, tesis de doctorado leida en la Universidad de Alicante el cursos 1996-1997, p. 860 , donde se encuentra el texto en que aparece la palabra). 
mos a encontrar: “...trobaren fita y contrafita, designant la fita, la guia del assagador de Consell y la contrafita, lo assagador de herbatge", (ibidem: 146).

En el siglo XIX, en el documento denominado Llibre de Visures de Tírig de 1817 que se encuentra en el Archivo Municipal de Tírig, lo encontramos de nuevo profusamente documentado con ejemplos de topónimos y de apelativos:

Dia 25 de Junio 1817, anaren a regoneise el Asagador del Barranch d'en Chaume, Sebastián Sentelles, procurador general... (Beltran Zaragozá, 1998, I: 147-177),

Ytem y pasan, al cantó de la Foya y a sen y quinse pases, endret de un.atra, a la part de Clemén Prats en trenta pases de amplària y quede achuntat a l'Asagador de la Ombrieta, (ibidem),

Ytem travesan l'Asagador dels Chimens, dret al Pla de l'Om, a les sen sincuanta pases trobaren una parella de creus, una a cada part en coranta pases de amplària y les varen abonar, (ibidem),

Ytem pasan per avan, travesan l'Asagador del Povacho y a tresentes y vuitanta pases de amplària feren una creu, (ibidem),

Ytem pasan, a noranta pases, al cantó de la Tancada de la Ombrieta trobaren fita y contrafita y atre costad, a vint-i-dos pases de amplària, feren una creu y travesaren el Asagador del Camí de Albocàser (ibidem),

Ytem abocan-se dret a l'Asagador de na Moragues y confrontan a l'Asagador del Camí de Alobocàser feren dos creus, una a cada costat, les dos en una seguida de clapises, en vin pases de amplària. Y se achunte a l'Asagador de na Moragues" (ibidem),

...pasan l'Asagador del Flaó y al cap de la figereta verdal, a la mà esquerra trobaren una fita parda y a la dreta, en dotse pases de amplària, en posaren un.atra... (ibidem),

Y pasaren de vesprada a l'Asagador de lo Barran del Puchol. Y comensan a les fites del Pou del Puchol que posaren lo dia tres de chuiñ (ibidem),

Ytem tornaren a l'Asagador del Pou del Mas d'en Runa. Encontraren dos fites en sincuanta pases de amplària y les tingueren per bones (ibidem),

Ytem baisan, a les sen seisanta pases, al cantó de entre Raymundo Albalad y Manuel Ballester feren una creu a cada part, en vint-i-set pases de ample y s'achunte a l'Asagador de la Costa Michana (ibidem)

Comensaren lo Camí de Santa Bàrbera eisín de l'Asagador de la Basa del Colomer (ibidem)

Ytem pasan per amun, crusan l'Asagador del Bovalar, a les sen vuitanta pases de ample, 67 del cantó de Chuan Segarra, a la heretad de Miguel Muntull (ibidem).

Todos los ejemplos que aparecen en este documento de 1817 están escritos con - $a$-. Los únicos casos que encontramos con - $e$ - son un topónimo y un apelativo añadido posteriormente al dicho documento: "Asegadoret de la Viñampla dret al Camí de Catí', (ibidem), "Tosalet (va per detràs l'asegador)", (ibidem). Parece que deben ser al menos de la segunda mitad del XIX. Hoy, la forma del nombre común en estas comarcas valencianas septentrionales es con -e-, como hemos tenido ocasión de comprobar (vide supra). 
La extensión geográfica del término es dilatada, como se ve, según autores y obras: Maestrat, la Plana de Castelló, Valencia (GGirona); Altea, Tárbena, la Vila Joiosa. Benidorm (Colomina); Calasseit, Maestrat, Castelló de la Plana, València (DCVB). En las comarcas castellonenses de habla catalana, tenemos lo siguiente: en el Alcatalén (Alcora) y en la Plana Alta (Vilafamés y Castelló de la Plana) distinguen entre la cañada ancha y entre localidades de la cañada estrecha y dentro del término municipal. En el primer caso se dice 'pas real' y 'segadoret' en Alcora. En el segundo caso, en Vilafamés un pas y carrerassa, y en Castelló de la Plana assagador y carrerassa. La toponíma rural nos da muchísima información sobre esta palabra. En Castelló de la Plana, tenemos el Assagador del Bovalar y el Camí de l'Assagador, (Gimeno-Arasa, 1993: 63). En Culla, existe el Assagdor de Castelló. Esta palabra es también usual (al lado de lligallo) en los Ports de Morella y el Alto Maestrat (Gimeno Betí, 1995: 87). El ALTA la recoge en Nonasp (vide mapa $\mathrm{n}^{\mathrm{o}} 310$ 'carrerada'). Nuestro ALDT es generoso con la extensión del número de localidades que lo recogen, nada menos que trece, bajo la variante assegador, que parece la más habitual de las hablas norteñas castellonenses. Respecto a la documentación literaria hay que decir que aparece en autores de la ciudad de Castelló: "...adreçà els pasos a la comanda de Garxolí per un assagador que a un altre menava, i aquest al barranc de la Parreta...", Josep Pascual Tirado, Tomba-Tossals, 1930, 57; "Alguns vells conten que ells encara han vist com a aquest pujolet acudien tots els assagadors del terme..." (apud DCVB, s.v.), Joan Porcar, Les cultures en la platja de Castelló, 82 (apud DCVB, s.v.).

Según Coromines proviene del latín secutorium (después de haber rehusado otras propuestas por él mismo y por otros autores, entre ellos F. de B. Moll), forma neutra del adjectivo secutorius, $-a$, -um 'el que viene detrás', que habría pervivido en mozárabe (DECLC, VII: 585). ( $c f r$. también REW 7778). Para el origen aragonés, vide DCELC, I: 346.

\subsection{GANADO (cat. y aragonés bestiar)}

El ALDT recoge los vocablos ganado y bestiar junto a ramat en el mapa num. 348 'El ramat' (esp. 'El ganado'). En este mapa, además, aparece recogido el término rabera ('idem), arabismo moderno en valenciano y tortosino.

Respecto a la palabra ganado, la hallamos documentada en textos latinos desde el siglo XI. Du Cange afirma que es un término propio de Hispania ("Ganata. ex Hispanico Ganato", 'Grex') especialmente aplicado a los animales de gran tamaño como bueyes y caballos, principalmente ("armentum"), y muy abundante según este diccionario en documentos hispánicos ( $c f r$. GMIL, IV: 22). Aparece ya en Las Glosas Emilianenses latinizado bajo la forma ganato. Ya en castellano, ganado se documenta en el siglo XI (1074) pero con el significado primitivo de 'ganacias', 'bienes'. En el poema del Cid (s. XII) aparece ya con el significado actual ( $c f r$. DCECH, s.v. ganar; vide además DME, II: 1186). La documentación que aportamos en este trabajo la recoge en latín, castellano y catalán. El documento más antiguo que lo atestigua es un texto en latín de 1327, datado en Morella:

pascerent ganata sua ganata sive bestiaria bestiaria sive ganata ganato ablato (Sánchez Adell, 1986: 321). 
Aparece también en un documento de 1341, en Teruel:

ganata sive bestiaria sua pascere

ganata sive bestiaria

sua ganata pascebant

bestiaria sive ganata (Sánchez Adell, 1986: 323)

En ese documento de Teruel de 1341, se recoge también en castellano como sinónimo de bestiar, que sería la forma aragonesa (Borao, 1859: 127):

paxer sus ganados si quiere bestiares

los dichos ganados

los ganados montados (Sánchez Adell, 1986: 324.45)

Un año después, en 1342, es documentado en un texto procedente de Daroca: "ganados" (Sánchez Adell, 1986: 328.5 i passim). No obstante, debemos esperar hasta 1390 para documentarlo en un texto catalan:

ganado o bestiar

lo bestiar o ganado

número de bestiar o ganado (Revest, 1957: 229.14).

El laude arbitral que publicó Don Luís Revest como apéndice a la edición del Libre de Ordenacions de la vila de Castelló de la Plana es el único documento de esta época en tierras castellonenses y tortosinas en que aparece la forma ganado, castellanismo muy antiguo $^{7}$ (vide supra) que penetró en catalán muy probablemente a través del aragonés (cfr. Colón, 1960a: 289; Gimeno Betí, 1995: 85). Parece que el único documento "literario" que registra este castellanismo es un Sermón de sant Vicent Ferrer:

¿E és deguna cosa que sie més profitable que Déus? No, car vehets vosaltres llauradors que de un gra que sembrau vos en done cent. Ell vos creix tots los béns: camps, vinyes, ganados, e tot vos ó done, e tot ó multiplique ${ }^{8}$ (ENC, III, p. 185.32; cfr. també Schib, 1977).

Finalmente, debemos referirnos al sintagma latino ganato ablato (que aparece en un documento de 1327 datado en Morella) a través del cual el texto se refiere al ganado sustraído: "vulumus juramento et juxta id restitucionem eis fieri de ganato ablato tatxacione judiciariua precedente" (Sánchez Adell, 1986: 321.31).

Respecto al término bestiar, palabra habitual en catalán y aragonés para referirse al conjunto de animales de pasto ${ }^{9}$, hay que decir que es la forma general en catalán y en todos los documentos analizados de esa época en estes tierras, a excepción hecha del caso de ganado mencionado arriba. El Du Cange también lo registra como forma sinónima de bestiare 'pecus' (GMIL, I: 645), esta, documentada en los Fueros de Huesca

\footnotetext{
${ }^{7}$ No hay ningún mozarabismo "rebrotado", como opinaba J. Coromines (DECLC, I: 784, nota 10); se trata de un claro castellanismo penetrado en el catalán de Valencia a través de los contactos con la ganadería trashumante castellana (Cuenca, sobre todo) y aragonesa (vide Gimeno Betí, 1995, y Sánchez Adell, 1986, entre otros).

${ }^{8}$ Información que debo a la amabildad de Germà Colón.

${ }^{9}$ Según Borao, sólo se aplica al ganado mular y caballar (1859: 127).
} 
(año 1247), y que parece ser la habitual en aragonés antiguo, al lado de bestiar (ut supra). Hay que añadir que, igualmente, el Du Cange remite bestiare a los términos bestagium y bestale, sinónimos de las formas mencionadas (cfr. GMIL, I: 644). Por otra parte, en estos documentos aparece también el término ramat como sinónim de bestiar: “...li ha furtades VII cabeces de ovelles les quals al dit en Matheu foren faltejades del seu bestiar per lo herbejador del dit comanador, e les quals diu que furtà e s.en menà del ramat o bestiar..." (Sánchez Adell, 1992-93: 385.7, año 1386),

Aunque en otro documento queda bien clara la diferencia entre ellos: “... an donada per justa una pena o penyora que los guardadós d'aquí havien feta de un moltó de un ramat de bestiar de hòmens d'Alcalà..." (ibidem: 383.3, año 1384).

\subsection{LIGALLO (cat. lligallo):}

El ALDT recoge el vocablo en el mapa núm. 203 'El camí ramader' (esp. 'cañada', 'camino de cabaña') y ocupa una mayor extensión que assagador puesto que se recoge en 18 localidades, que son de norte a sur: Rasquera, Benifallet, Paüls, El Perelló, Tortosa, Mas de Barberans, Alcanar, Ulldecona, Sant Joan del Pas, Els Valentins, La Sénia, Sant Rafel, Canet lo Roig, El Boixar, Fredes, La Pobla de Benifassà, Pena-Roja de Tastavins y Beseit.

El vocablo "lligallo" es el apelativo general que se da en tortosino al camino que tienen derecho a seguir los pastores con sus ganados cruzando propiedades. El diccionario Alcover-Moll lo hace sinónimo de cañada; propio de Tortosa. Efectivamente, ya hemos visto su extensión geográfica (vide supra). J. Coromines afirma que es común con el bajo-aragonés y de origen mozárabe (DECLC, V: 208; cfr també Colón, 1960: 290). Documentado a partir del siglo XIII como una institución jurídico-agropecuaria, hoy queda en esta zona como sinónimo de cañada (DECLC, V: 208 i cfr també Veny, 1991: 111). Este nombre común aparece ya como topónimo, por ejemplo, en Gandesa: La Carrerada del Serrall del Lligalló, con pronunciación oxítona, así como también en Benissanet: Barranc del Lligallo (Gimeno Betí, 1991: 629-630). Proviene de una forma latina *LIGACULUM con U breve, que es un derivado de LIGARE ( $c f r$ DRAL, s.v.). La forma ligallo se puede explicar lingüísticamente desde la óptica aragonesa . La L- inicial no palataliza, generalmente, en aragonés, aunque hacia la región oriental, Ribagorza y parte del Sobrarbe, hay casos de palatalización actualmente, influencia posible del catalán. El grupo románico C'L en la lengua antigua estaba representado por $l l$, articulación que todavía hoy es la más representativa del dialecto, frente a la consonante velar castellana $/ \chi /$. Finalmente, la -o final de palabra se comporta en aragonés de una manera difícil de definir. Entre el catalán que la elimina, y el castellano que la conserva, el aragonés parece la zona de tránsito natural; es decir, igual se conserva que se elimina, aunque el mantenimiento parece que sea la tónica dominante (Zamora Vicente, 1974: 220, 224 i 244). Por otra parte, podemos afirmar que, fuera del ámbito ya estrictamente lingüístico, hay circunstancias concretas que han ayudado a la penetración en catalán de léxico aragonés como por ejemplo el comercio ganadero y el pastoreo, que explicarían esta penetración en valenciano septentrional y en tortosino de palabras como nuestro lligallo y otras de ese ámbito. El Diccionari Aguiló recoge una forma lligall que parece no estar documentada en ninguna parte (IV: 315). El Alcover-Moll ya recoge la forma con -o final y la sitúa geográficamente, como ya se ha visto, solamente en Tortosa. Es voz recogida también por Pardo Asso (1938, s.v.) con el significado de "junta de 
ganaderos o reunión anual de dueños y pastores en que antiguamente se dirimían las controversias sobre el paso de ganados", acepción que copia del DRAL. Este diccionario la sitúa geográficamente en Aragón.

En cuanto a la documentación de la palabra en valenciano, tenemos, en primer lugar, la creación del tribunal del "lligallo" de Morella. El término aparece por primera vez en 1271 en texto latino que hace público este tribunal, publicado por Valls Taberner $^{10}$, junto a otro, también latino, de 1316 . En catalán se documenta a partir de 1329:

Atenen encara que si $\cdot 1 \mathrm{~s}$ dits bestiars estranys, de muntada o devayllada, no cascaven o barien la dita partida de terme, que serie més profit e utilitat a les rendes de la senyora Regina en ço que molts bestiars del terme de Morella que parexen fora del terme, dels quals no és aguda a senyor sinó miga delma, aturarien e parien qui és acostumat de fer en Morella, al qual lligallo se menen e a menar se deuen los dits bestiars... (Sánchez Adell, 1992-93: 371).

Vuelve a documentarse (cuatro veces) en un texto de 1341 datado en Valencia. Se trata de una sentencia favorable al monasterio de Benifassà, de Domingo Monçó, juez delegado por el rey, en el pleito entre la villa de Morella y el monasterio a causa del Lligallo:

E vista la pronunciació feyta per lo dit Balle et manament feyt ensemps ab en Bernat Pinós et en Johan Sanç, justícies del lligallo, et la letra per aquells manada fer (Sánchez Adell, 1995: 480-483),

... sie atorgat al consell de Morella et a tots los hòmens de les aldeyes et de tots los lochs et térmens de aquella, axí del senyor rey com de órdens, com de cavallers, qualsevol que poguessen fer lligallo en lo terme de Morella, (ibidem),

Emperò no appar que.ls sobredits haien ordenat lo dit lligallo ne que aien ordenat en qual loch del terme de Morella... (ibidem)

En 1358, existe el documento de concesión a las localidades castellonenses de Càlig, Canet lo Roig, Rossell, la Jana y la Barcella para poder ejercer cada año justicia del Lligallo, en que la voz aparece profusamente citada:

... que comunament cascun any en lo loch de la Jana, tots temps poguessen fer e tener ligallo de moltes e diverses bestiars que van exarrats e radius... (Díaz Manteca, 1987: 263-264), ... axí en los térmens dels dits lochs com en altres circumvehins, com tenir lo dit ligallo sia gran profit de les dites universitats... (ibidem),

Attenents res no menys lo dit loch nostre de la Jana ésser suficient e convinent a tenir e celebrar lo dit ligallo..., (ibidem),

... dels damunt dits lochs de Canet, de Rossell, de la Barcella, de la Jana e de Càlig, presents e esdevenidors, que puxen fer, tenir e celebrar cascun any ligallo en lo dit loch de la Jana... (ibidem),

Primerament: que.l dit ligallo sia fet en lo dit loch de la Jana, d'ací avant, és a saber, lo dilluns immediat segunt aprés de la festa de Pascha de la Ressurrecció...(ibidem),

Continúa la documentación en 1375, ahora en latín, dos veces:

\footnotetext{
${ }^{10}$ Este autor convirtió equivocadamente la palabra en oxítona (lligalló), cuando en realidad se trata de una forma paroxítona (lligallo), puesto que es un aragonesismo como podemos ver. El primer filólogo que lo hizo notar fue G. Colón (1960: 290).
} 
Pro parte Abbatis et conventus Monasterii de Benifaçano fuit nobis expositum querelosa quod licent homines locorum tenentie dicti Monasterii nunquam ubi fuerint ire ad faciendum Ligallo... (Sánchez Adell, 1992-93: 375 i 1995: 483),

Vos tamen pridem precepto penali injuncxistis hominibus predictis jam dicte tenencie quod irant pro tenendo Ligallo... (ibidem). tar:

Un texto del año siguiente (1376), escrito también en latín, lo vuelve a documen-

Tamen quia nos de causa seu questione que inter partes jam dictas seu etiam procuratorem nostrum fischalem ventilatur pretextu convocationis sive cuisdam juris lo Ligallo vulgariter nuncumapti sine tamen lesione... (Sánchez Adell, 1995: 484-485),

Stratim vero et ante omnia vos informetis sumaria si officiales nostri sunt in possessione pignorandi pro dicto dicto jure del Ligallo... (ibidem)

Otro texto donde encontramos la palabra es un breve documento de 1384; se trata de una orden hecha al justicia del lligallo de Cervera del Maestrat para que devuelva ganado, donde la palabra aparece tres veces:

De part del Maestre de Muntesa al feel e amat nostre lo justícia del ligallo de la vila nostra de Cervera o son lochtinent, saluts e dilecció. Diu en Miquel Calbo, de la vila nostra de Sent Matheu, que ell ha trobades, en los bestiar del vostre ligallo, una ovella e una borega sues, que no li volets donar aquelles... contrastats sens justa raó, perquè a suplicació de aquell consonant aró, volem e a vos dehim e manam que vista la present donets e liurets al dit en Miquel les dites ovella e borega, ell jurant que són sues, e pagan ço que.ls altres que traen bestiar del dit ligallo paguen, o demà disapte siats davant nos..." (Sánchez Adell, 1992-93: $383)$.

Podemos señalar un texto de Castelló de 1390 como el documento inmediatamente posterior cronológicamente:

Item dehim e pronunciam que cascuna vegada que.s farà lligallo en cada sesma o aldeya de Terol, segons és acustumat, tots los majorals de les cabanes dels hòmens e vehins de Castelló que seran ab los seus bestiars en aquella cesma o aldeya on se farà lo lligallo sien tenguts venir al dit lligallo e a dur totes les mostrengues que en los seus bestiars tendran les quals sien tengudes restituhir al dit lligallo e fer totes les altres coses que antigament són acustumades fer en lo dit lligallo per los vehins propris de aquella aldeya et de les dites ciutat e aldeyes. Et per semblant manera los vehins de les dites ciutat e aldeyes sien tenguts venir a tot ligallo que.s farà en lo terme de la dita vila de Castelló si seran presents en la vila $\mathrm{e}$ terme de aquella e a dur e restituhir totes les mostrenques e fer totes les altres coses que són acustumades per los vehins de la dita vila... (Revest, 1957: 23-235)

Otro documento que recoge el término lligallo, referido a la institución ganadera, es el Llibre d'Establiments de la vila de Culla del año 1593, en el qual aparece la palabra, como en el documento anterior, citado profusamente (vide Rabassa, 1992: 93-94). Este libro incluye un apartado que lleva como título Rúbrica del ligallo donde aparecen una serie de mandatos dirigidos al buen regimiento de aquella institución, y en el qual el vocablo se documenta veintitrés veces, y además remite a unas posteriores ediciones de las rúbricas de los años 1716-1718, que según Rabassa fueron redactadas en 1718. Es decir, la palabra se registra también en los siglos XVI y XVIII.

La influencia del aragonés sobre el catalán respecto al mundo de la ganadería es importante y viene de lejos ( $c f r$. Veny, 1991: 110). Por otra parte, podemos constatar 
que las relaciones entre Aragón y el País Valenciano a causa de la ganadería están documentadas también desde la edad media ( $c f r$ Sánchez Adell, 1980: II: 821-826 y 1982: 113-124; vide también Gimeno Betí, 1995). Debemos recordar que antes de la creación del Reino de Valencia por Jaime I, las comarcas castellonenses ya conocían un tráfico de ganado entre el Aragón cristiano y el territorio valenciano todavía musulmán; existía por tanto una trashumancia entre aquellas tierras. En 1271 se concede a Morella y sus aldeas el derecho de "facere ligallum", es decir, a constituirse en la institución del "lligallo", que tenía por objeto reunirse dos veces al año para tratar la devolución y posterior repartición de las cabezas de ganado sin dueño que, en caso de no aparecer, se custodiarían hasta la próxima reunión (Hinojosa, 1980: 182; Milián, 1927: 179). Este tribunal sancionó pleitos y problemas ganaderos hasta 1855, año en que fue abolido por una real orden (Milián, 1927: 185). El ligallo era una institución aragonesa antigua que databa de 1259 ( $c f r$. Veny, 1991: 110). Tenemos noticias de ella también en Segorbe, Càlig, Canet lo Roig, Rossell, la Jana y la Barcella (Bajo Maestrazgo) en 1358 al igual que en Tortosa (vide supra la documentación). Este tribunal morellano estuvo muy relacionado a partir del siglo XIV con el de Tortosa a través de la mutua ayuda que se prestaban en determinados casos (Milián, 1927: 181). Todos estos antecedentes, así como otros que no mencionaremos aquí (cfr Obiol, 1989: 231), denotan una intensa actividad ganadera dentro del espacio rural medieval de Castellón. La ganadería, fuente principal de la riqueza de la comarca del Bajo Maestrazgo y también de los Ports de Morella con sus carnes, lanas y cueros, contribuyó a estrechar más las relaciones entre las tierras aragonesas y las valencianas del norte. A les dehesas de Vallivana, Turmell y otros prados comunales de aquellas comarcas se trasladaban los ganados aragoneses a invernar (Betí, 1927: 98).

\subsection{MALLADA}

El ALDT recoge el término en el mapa núm. 386 'Excrement de conill' (esp. 'Excremento de conejo'), pero por lo que veremos más abajo no concuerda semánticamente con la definición que nos da de él el diccionario de Alcover-Moll. En nuestro atlas solamente se ha recogido en la localidad de Rasquera.

Respecto a la palabra mallada 'lloc arrecerat on s'aturen a reposar els pastors i llurs ramats' (DCVB,VII: 172), debemos decir que el vocablo solamente aparece en tres documentos del siglo XIV de estas comarcas, que conforman la variedad diatópica tortosina $^{11}$, dos de los cuales están datados en 1382, tres años antes, pues, que el citado por el Alcover-Moll, y que reproduce Coromines en su diccionario etimológico catalán, y el otro en 1389. Son los siguientes:

...axí mateix e son pare e los seus predecessors tenir aquí de nit e de dia mallada..., (Sánchez Adell, 1992-93: 378.24, documento datado en Fanzara en 1382);

... no acostumaren de tenir ni pasturar los seus bestiars en lo dit terme e devesa de Fanzara ni tenir aquí mallada..., (ibidem, 378. 32);

\footnotetext{
${ }^{11}$ Hay que añadir el término mallada está documentado con anterioridad a estos diplomas, en 1345-1370 (Barreda, 1986:472-476).
} 
Et axí mateix que jamés null temps d.ací avant no volie ni entenie que ell ni los seus successors del seu mas o ostal tinguessen ni fessen ni poguessen fer o fer fer per alcuna manera de nit mallada dels lurs bestiars..., (ibidem, 378.53);

... no faran, ni tindran, ni fer faran, ni tenir volen, mallada dels dits seus bestiars en lo terme e devesa de Fanzara..., (ibidem, 379.12)

Et parteix d.aquí et passe lo camí que va del dit lloch de Fredes a Mallada del Magraner et als molins de Benifaçà, (Sánchez Adell, 1992-93: 382.23, documento datado en el Monasterio de Benifassà en 1382)

Primerament, prengueren per a mallada una cova d'en Domingo Melià, la qual és al cap de la sua heretat $[\ldots]$ e afronte, la dita, mallada ab la heretat que ere antiguament d'en Osca [...] Item, prenguem mallada, ço és un buschal d'en Berthomeu Ezvalls, lo qual afronta [...] e aquí havem camí que mou de la cova e mallada e plegue al pou que és appellat d'en Borraç [...] e aquí morí camí qui va per lo barranch avall entrò al terme de Tírig, segons que és ficat, los quals camins e abeuradors e mallades eren ia antiguament de consell [...] Item, prenguem mallada una cova d'en Johan Mercer al sol del Barranch del Milà [...] Item, havem una cova e mallada, que és appellada d'en Codera ab gran pati e many del camí, que va a Senct Matheu [...] Item, prenguem per a mallada en la heretat d'en Domingo Fort [...] Item, havem camí que mou de la dita mallada per lo barranch avall tro a la Font del Bosch [...] Item, havem camí de XXXX palms que mou de la Corriola, la qual va a la mallada que.ns havem presa d'en Domingo Fort [...] Item, prenguem mallada en la heretat d'en Pere Torner en un solanet damunt lo buschal de la terra roja amunt..., (Díaz, 1987: 440-442, documento datado en Albocàsser en 1389).

Según parece, el vocablo proviene del latín *MACULATA 'red que forma el redil' (cfr. DCELC, III: 196, etimologia admitida por el Alcover-Moll y per Rohlfs, 1977: § 259), no obstante habría pasado al catalán a través del aragonés, procedente del castellano (vide DCELC, III: 196-197) ${ }^{12}$. Actualmente, es forma todavía viva en catalán y aragonés, a través de la toponimia, en la zona central de la lengua catalana, sobretodo (comarcas catalanas meridionales y valencianas septentrionales; $c f r$. O.C., V: 154 y DECLC, V: 397-398). En el ámbito románico, aquella forma latina ha dado los siguientes resultados: catalán mallada, castellano majada, aragonés mallata, portugués malhada i gascón malhade (vide DCELC, III: 196-197, REW, 5212 y Rohlfs, 1977 § 259). En castellano, ya está documentada con este significado desde finales del siglo XII (a. 1182), y aparece en textos literarios primerizos (Berceo). En portugués, es una forma atestada desde el siglo XIII (DELP, IV: 32) con el significado de choça 'cabaña' (vide también, para el significado que aquí tratamos, DCELC, III: 196) ${ }^{13}$. Entre los derivados de la palabra mallada, tenemos en estos diplomas el intensivo malladar 'cueva en la montaña, a propósito para hacer mallada en ella y reposar el ganado’ (DCVB, VII: 172)

\footnotetext{
${ }^{12}$ No todos los autores entán plenamente de acuerdo en que la palabra mallada sea un castellanismo; por ejemplo, G. Colón afirma que el plural de mallada, con la variante mellades - que podemos leer en un documento de 1414 en el libro Epistolari de la València Medieval (II) d'Agustí Rubio Vela- quizá sea castellanismo. (cfr. G. Colón, 2000: 49).

${ }^{13}$ J. Coromines critica un étimon ("no tiene verosimilitud") MALIATA -según él no documentado- propuesto para el caso en la nota 4 del artículo majada del DCEC, sin reparar que el Du Cange recoge las formas latines MALLIA, MALLATA y MALLIATA ( $c f r$. GMIL, IV-V: 197 i 199).
} 
que este diccionario sitúa en Tortosa, sin ninguna cita documental. Borao también recoge la forma verbal amalladar (1859: 113). He aquí el pasaje donde aparece el apelativo, convertido en topónimo: “... et parteix d.aquí et dóne tot coyll avall, axí com aygües vessen, envers lo dit lloch de Fredes per cap de la ombria sobirana segons les roques tayllen, et dóne al Malladar d.en Poma", documento datado en el Monasterio de Benifassà en 1382 (Sánchez Adell, 1992-93: 382.15).

\subsection{ARABOGA:}

El ALDT recoge el término en el mapa num. 193 'roina' (esp. 'llovizna') en les localidades castellonenses de Sorita, en la comarca de los Ports de Morella, Les Coves de Vinromà y Vilanova d'Alcolea, las dos de la comarca de la Plana Alta. Joaquín García Girona, en su Vocabulari del Maestrat, definía así el término: "Fan arabogues, intermitències de pluja menuda i breu. Arabogues de neu; quan son de neu trida i a vegades mesclada en pluja". Aparece en los repertorios lexicográficos del catalán occidental (norte-occidentales, valencianos y tortosinos): Massip (1991: 297) arabogues 'pluja intermitent i menuda: quan fa vent i el vent porta nuvolades i cauen quatre gotes; al cap d'unes hores un altre núvol, i torna a ploure etc'. Está documentado en los diccionarios históricos catalanes: DCVB (I: 808) araboga 'pluja fina d'aigua mesclada amb neu', 'intermitències de pluja menuda i breu'; DECLC (I: 103-104) araboga, la misma definición que Girona. La toponimia també recoge el nombre: el Mas d'Arabogas entre la Todolella y Olocau. No es registrado por el Diccionari de la Llengua Catalana (IEC). Su extensión geográfica es la siguiente: Maestrat (GGirona); Morella, Benassal, Alcalà de Xivert (DCVB), Benassal (DCVB), Portell de Morella (DECLC). Es un vocablo vivo en las comarques castellonenses del Alcalatén (l'Alcora, Xodos) araboga 'pluja fina', (Llucena) arabogà, Alto Maestrazgo (Culla) arebogues, (Tírig ) arabogues son 'fortorades de fred, aigua de pluja amb un ruixat que va mesclat amb vent', en el Bajo Maestrazgo (la Jana) arabogues 'pluja curta i amb poca quantitat mesclada amb vent fred', (Salzedella) arabòc 'núvol solt, amb vent fort, que descarrega una ramalada d'aigua $\mathrm{i}$ se'n va', en los Ports de Morella (el Forcall) araboga 'pluja fluixeta'. También conocido en las comarcass castellonenses de habla castellano-aragonesa: Sucaina: araboga 'aguanieve'. En la Plana de Castelló, tiene diversas denominaciones: torrelló (Vilafamés, Bell-1loc), araboga (la Torre dels Domenges, Orpesa, Aín).

Literariamente, se documenta en "Lo cap nevat de Penyagolosa nunci de la freda araboga que l'Aragó ens aventa", Gaetà Huguet, BSCC, III: 156 (apud DCVB, s.v.); “... fea fred i el temps amenaçava arabogues", Bolangera de Dimonis, 52; "I per aquest temps -dia- les arabogues arrematen en tempesta", Bolangera de Dimonis, 52; "Les arabogues que començaren en eixir de Sorita s'han tornat pluja forta, ...”, Bolangera de Dimonis, 53; "¿I qui no ha conegut un dia de sant Antoni d'arabogues, o un dia tot nevat...?", Carles Salvador, Les festes de Benassal, 20; "No sabem si aquesta nit fa bona hora o fa vent o neva, o si les arabogues mullen les mantes", Carles Salvador, Les festes de Benassal, 39.

Etimológicamente es un derivado del latín aer. Como compuesto podría venir de àer, ar, o de su plural aera, pero el problema proviene a la hora de explicar el segundo componente -boga. El mismo Coromines no lo ve nada claro y propone dos etimologías: $1^{\mathrm{a}}$ àer a voga 'aire veloç, que corre com una nau a voga tirada', $2^{\mathrm{a}}$ un compuesto mozárabe arabizado aera pluvia > pre-arábigo a(e)-raploia arabizado en *arabroya, 
disimilado en *araboya y a causa de la repugnancia del árabe para la combinación $u y$ cambiando en arabû(w)a > araboga ( $c f r$. DECLC, s.v.). Para una nueva propuesta etimológica se debe consultar Colomina (1995: 113 y nota 17) donde se afirma que araboga ha de ser una feminización de araboc ( $<$ arreboc $<$ arrebocar), si no es que provenga de la influencia de palabras como por ejemplo rebugada 'brossa arremolinada i aturada a un lloc pel corrent d'un xaragall o torrentada' y rebugall 'residu' (DCVB, s.v.). En aragonés, generalmente, significa 'tormenta o chubasco breve'.

\section{Bibliografia}

Aguiló I Fuster, Marià (1914-1934) Diccionari Aguiló. Materials lexicogràfics aplegats per M. Aguiló i Fuster. Revisats i publicats sota la cura de Pompeu Fabra $i$ Manuel de Montoliu. Ed. Altafulla, Barcelona [= DAg].

Alcover, Antoni Ma i F. de B. Moll (1975) Diccionari català-valencià-balear. Segona edició, corregida i posada al dia. 10 vols. Palma de Mallorca. [= DCVB].

Alonso, Martín (1986) Diccionario medieval español. Desde las Glosas Emilianenses y Silenses (s. X) hasta el siglo XV, I-II. Universidad Pontificia de Salamanca. Salamanca [= DME].

Alvar, Manuel (1953) El dialecto aragonés. Gredos, Madrid.

BARREDA I EDO, Pere-Enric (1999) "Els Establiments de la vila de Benassal. Introducció, resum, edició i índexs per Pere-Enric Barreda, Cronista Oficial de la Vila", dins Cooperativa Agrícola Benasalense. 50 aniversari. 1949-1999 (II), edición de la Cooperativa Agrícola Benasalense, pp. 5-160. Benassal.

BEC, Pierre (1968) Les interférances linguistiques entre gascon et languedocien dans les parlers du Comminges et du Couserans. Essai d'aréologie systématique, París.

Beltran Zaragoza, Andreu (1998) El valencià al segle XVIII. Edició $i$ estudi de textos administratius de les comarques del nord de Castelló, 2 volums. Societat Castellonenca de Cultura, col.lecció "Filologia, II". Castelló de la Plana.

Betí BonfiLl, Manuel (1927) "Las cruces gemelas de San Mateo y de Linares de Mora" Boletín de la Sociedad Castellonense de Cultura, VIII, (III), 97-109.

Blanc GraU, Miquel (1994) Garba. Mil paraules de Calaceit. Columna. Barcelona.

BORAO, Gerónimo (1859) Diccionario de voces aragonesas. Zaragoza.

CASACUBERTA, Josep Ma \& Joan COROMINES (1936) "Materials per a l'estudi dels parlars aragonesos. Vocabulari”, Butlleti de Dialectologia Catalana, 158-183. Barcelona.

CASANOVA, Emili (1989) "La faceta lexicogràfica del pare Fullana", Boletín de la Sociedad Castellonense de Cultura, LXVII, (III), 415-441.

COLOMINA I CASTANYER, Jordi (1991): “Joaquim Martí Gadea com a lexicògraf i com a dialectòleg", Caplletra, 11, 147-166.

- (1995) Els valencians i la llengua normativa.. Generalitat Valenciana. Conselleria d'Educació i Ciència. Institut de cultura "Juan Gil-Albert" (Diputación de Alicante). Textos Universitarios.

Colón, Germà (2000) "Sobre la lengua del Epistolari de la València medieval (siglo XV)", dins Revue de Linguistique Romane no 253-254, Janvier-Juin 2000, pp. 4351.

- (1960), recensión a Libre de Ordinacions de la Vila de Castelló de la Plana. Estudio preliminar, edición, notas y glosario de Luis Revest y Corzo. Castellón de la Plana, 
Sociedad Castellonense de Cultura, 1957, 323 pp., 18 x $26 \mathrm{~mm}$. Zeitschrift für Romanische Philologie, 76, 1960, pp. 285-291. Max Niemeyer Verlag. Tübingen.

- (1968) «Catalán enemic, aragonés enemigo, alemán Neidnagel», en Kurt Baldinger (ed.), Festschrift Walther von Wartburg zum 80. Geburtstag. Tübingen: Max Niemeyer, 1968, vol. II, pp. 415-420. Reeditado en El léxico catalán en la Romania, pp. 309-315.

COROMINAS, Joan (1955-1957) Diccionario crítico etimológico de la lengua castellana. Gredos, Madrid, 4 vols. [= DECLC]

Corominas, Joan \& Juan Antonio Pascual (1980-1989) Diccionario crítico etimológico castellano e hispánico, I (A-CA) 1980; 3 ${ }^{\text {a }}$ reimp. 1991, 938 p.; II (CE-F) 1980; $2^{\text {a }}$ reimp. 1989, 985 p.; III (G-MA) 1980; $2^{\text {a }}$ reimp. 1989, 903 p. [= DCECH]

Coromines, Joan Onmasticon Cataloniae, I (1989) Toponímia antiga de les Illes Balears. Joan Coromines i J. Mascaró Passarius; II (1994) A-BE; III (1995) BI-C; IV (1995) D-J; . Curial Edicions catalanes, Caixa de Pensions "La Caixa". Barcelona. [= OC].

- (1977) Entre dos llenguatges, III. Curial. Barcelona, 119.120.

- (1980-1991) Diccionari Etimològic $i$ Complementari de la Llengua Catalana. 9 volums. Barcelona [= DECLC].

Cuc, Toni de (1995) En Castelló, carabasses al balcó. Ajuntament de Castelló de la Plana.

DíAz MantecA, Eugenio (1987) El "Libro de Poblaciones y Privilegios" de la Orden de Santa María de Montesa (1234-1429). Diputació de Castelló de la Plana.

Diccionari De La Llengua CATAlana (2005), Institut d'Estudis Catalans. Barcelona.

ElCOCK, W. D. (1937) De quelques affinités phonétiques entre l'aragonais et le béarnais, París.

ESCRIG, José (1851) Diccionario valenciano-castellano .Dedicado á la Sociedad económica de amigos del país de esta ciudad de Valencia. Servicio de reproducción de libros. Colección Biblioteca Valenciana. Librerías "Paris-Valencia". Valencia, 1979, 2 vols.

ESTEVE, Joan (1489) Liber Elegantiarum. Estudio preliminar por Germán Colón Doménech. Inculca. Castelló de la Plana, 1988.

Foguet Mateu, Manuel (1984) Bona nit, cresol. Comedia en dos actos y un epílogo. Biblioteca d'Autors Vinarossencs. Associació cultural "Amics de Vinaròs". Vinaròs.

Frago, Juan Antonio (1977) "Sobre el léxico aragonés. Datos para el estudio de su frontera con el catalán noroccidental a mediados del siglo XV", Actes del Cinquè Col-loqui Internacional de Llengua i Literatura Catalanes, Abadia de Montserrat, pp. 403-440.

GARCIA GIRONA, Joaquim (1920) Seidia. Impremta Valencianista.València.

- (1922) Vocabulari del Maestrat. Societat Castellonenca de Cultura. Castelló de la Plana.

GARCIA PERAles, Vicent-Ferran (1995) Atlas Lingüístico de la Península Ibérica (ALPI): Edició i estudi del País Valencià. Memòria de Llicenciatura. Universitat de València.

GIMENO BETí, Lluís (1995b) "L'adstrat aragonès en un document castellonenc del segle XIV", dins Llengües en contacte als Regnes de València i Múrcia (segles XIII$X I V)$, Jordi Colomina i Castanyer (editor), Universitat d'Alacant. Departament de Filologia Catalana, "Biblioteca de Filologia Catalana" Alacant, 79-98. 
GIMENO BETí, Lluís \& Ferran ARASA GIL (1993) La toponímia el terme municipal de Castelló de la Plana. Ajuntament de Castelló de la Plana, 154 pàgines +31 plànols de situació dels noms de lloc.

- (1991) "Ornitonímia castellonenca: notes onomasiològiques i etnogràfiques sobre el nom d'alguns ocells", Boletín de la Sociedad Castellonense de Cultura LXVII, (III), 589-614. Castelló de la Plana.

- (1997) Atles Lingüistic de la Diòcesi de Tortosa. Institut d'Estudis Catalans. Biblioteca de Dialectologia i Sociolingüística, IV, 700 pàg. Barcelona [= ALDT].

Glossarium Mediae Et Infimae Latinitatis, conditum a Carolo du Fresne Domino du Cange, Niort 1883-1887, 10 vols., I (A-B), II-III (C-F), IV-V (G-N), VI-VII (O-S), VIII-X (T-Z). Reproducción fotomecánica de Akademische Druck-U. Verlagsanstalt. Graz-Austria, 1954 [= GMIL].

GRAN ENCICLOPEDIA ARAGONESA

HinOJOSA, F. (1980) "La ganadería valenciana", Nuestra historia. Mas Ivars

LAMARCA, Luis (1839) Ensayo de un diccionario valenciano-castellano .Servicio de reproducción de libros. Colección Biblioteca Valenciana.València, 1980.

LlOMBART, Constantino \& José ESCRIG (1887) Diccionario valenciano-castellano. Obra dedicada á la Sociedad de Amigos del Pais de Valencia. Servicio de reproducción de libros. Colección Biblioteca Valenciana. Librerías "Paris-Valencia". Valencia, 1987, 2 vols. [= Llombart]

MACHADO, J. P. (1953-1959) Dicionario etimológico da língua portuguesa com a mais antiga documentaçao escrita e conhecida de muitos dos vocábulos estudados, 2 vols. Lisboa. [= DELP].

MARTÍ Y GADEA, Joaquín (1891) Diccionario general valenciano-castellano . Servicio de reproducción de libros. Librerías "Paris-Valencia". Colección Biblioteca Valenciana. Valencia, 1992, 2 vols.

MARTí I MESTRE, Joaquim (1994): "Una contribució a la lexicografia del segle XVIII: Marc Antoni d'Orellana", Miscel.lània Germà Colón, 1. Publicacions de l'Abadia de Montserrat, 111-145. Barcelona.

- (1996) Col.loquis eròtico-burlescos del segle XVIII. Edicions Alfons el Magnànim. Institució Valenciana d'Estudis i Investigació. Biblioteca d'Autors Valencians / 36. Generalitat Valenciana. Valencia.

- (1997) Literatura de canya i cordell al País Valencià. Els col.loquis de temàtica jocosa $i$ satírica. Edició $i$ estudi lingüistic. Col.lecció Francesc Ferrer Pastor, Investigació, 2. Editorial 10 Denes. Valencia.

MASSIP I BONET, Ma Àngels (1991) El lèxic tortosí: història i present. Tesis de doctorado. Universitat de Barcelona.

MEYER-LÜBKE, Wilhelm (1972) Romanisches Etymologisches Wörterbuch. Fünfte Auflage. Heidelberg. Carl Winter-Universitätsverlag. [= REW].

MiLIÁN BOIX, Manuel (1927) "Notas para la historia del Lligalló de Morella", Boletín de la Sociedad Castellonense de Cultura, VIII, (IV), 179-185. Castelló de la Plana.

NAVArro GómeZ, Pere (1996b) Els parlars de la Terra Alta. 2. Atles Lingüístic de la Terra Alta (ALTA). Diputació de Tarragona. Tarragona. (= ALTA).

OBiol Menero, Emilio M. (1989) La ganadería en el norte del País Valenciano. Publicaciones del Excelentísimo Ayuntamiento de Castelló de la Plana.

PARDo ASSO, José (1938) Nuevo diccionario etimológico aragonés. (Voces, frases y modismos usados en el habla de Aragón). Zaragoza. 
Pascual I TIRADO, Josep (1930) Tomba-Tossals. Contalles de la terra. Societat Castellonenca de Cultura. Castelló de la Plana.

PERAIRE, Joan (1992) "Aproximació a la toponímia històrica de la Tinença de Culla (segles XVI-XVIII)", Actes del XVIè Col.loqui de la Societat d'Onomàstica. Castelló de la Plana, 1991. BISO, XLVIII (gener 1992), 115-157.

QUINTANA, Artur (ed.) (1995) Lo Molinar. Literatura popular catalana del Matarranya i Mequinensa. 1. Narrativa i teatre. Col·lecció Lo Trill (1). Instituto de Estudios Turolenses. Associción Cultural del Matarraña "Carrutxa". Calaceite.

RABASSA I VAQUER, Carles \& Carmen DíAZ DE RÁBAGO HERNÁNDEZ (1995) Documents per a la història de Vilafamés. Generalitat Valenciana/Consell Valencià de Cultura/ Ajuntament de Vilafamés/Universitat Jaume I. València.

REAL ACADEMIA ESPAÑOLA (1992) Diccionario de le lengua española, vigésima primera edición, 2 vols. Espasa-Calpe. Madrid.[= DRAL].

REVEST Y CORZO, Luis (ed., 1957) Libre de Ordinacions de la vila de Castelló de la Plana. Societat Castellonenca de Cultura. Castelló de la Plana.

RoHLFS, Gerhard (1977) Le Gascon. Études de philologie pyrénéenne. Max Niemeyer Verlag. Tübingen. Troisième édition, augmentée.

Salvador, Carles (1952) Les festes de Benassal. Biblioteca Folklòrica Barcino, III. Editorial Barcino. Barcelona.

SÁNCHEZ ADELL, José (1980) "Datos para la historia de la transhumancia ganadera castellonense en la baja edad media", Primer Congrés d'Història del País Valencià, II, pp. 821-826. Valencia.

- (1986) "Aportaciones a la historia de la ganadería medieval castellonense. La sentencia de Villahermosa entre Castellón y las aldeas de Teruel, sobre pastos, de 1390", Estudis Castellonencs, No 3, pp. 311-336. Castelló de la Plana.

- (1992) "Toponímia del Castelló medieval", Actes del XVIè Col.loqui de la Societat d'Onomàstica, a cura de Lluís Gimeno Betí \& Ferran Arasa Gil. XLVIII (gener 1992), 21-31. Castelló de la Plana.

- (1995) "Aportaciones a la historia de la ganadería medieval en las comarcas del norte castellonenses (Ports de Morella, Benifaça y Maestrazgo de Montesa)", Boletín de la Sociedad Castellonense de Cultura, LXXI (IV), pp. 445-487. Castelló.

SÁNCHEZ AlMELA, Elena (1985) El Llibre de Privilegis de la vila de Sant Mateu (11571512). Publicaciones del seminario de estudios económicos y sociales de la Caja de Ahorros y Monte de Piedad de Castellón. Castelló de la Plana.

SÁnchez GozALBO, Àngel (1979) Bolangera de dimonis, $3^{\mathrm{a}}$ edición especial para la Caja de Ahorros y Monte de Piedad de Castellón. Castelló de la Plana.

SCHIB, Gret (1977) Vocabulari de Sant Vicent Ferrer. Fundació Salvador Vives Casajuana. Premi "Gumersind Bisbal Gutsems, 1976". Barcelona.

VENY, Joan (1991) Mots d'ahir i mots d'avui, Empúries. Barcelona.

ZAMORA Vicente, Alonso (1974) Dialectología española, Gredos, Madrid. Segunda edición muy aumentada. 